\title{
Clinician perspectives on models of care for pediatric weight management services in Australia: A mixed-methods study
}

Jennifer Cohen ( $\square$ jennifer.cohen2@health.nsw.gov.au )

Sydney Children's Hospital Network https://orcid.org/0000-0002-7798-1113

Shirley Alexander

Children's Hospital at Westmead

Christina Signorelli

University of New South Wales

Kathryn Williams

The University of Sydney

Kyra A Sim

The University of Sydney

Lenina Chennariyil

Canterbury Hospital

Louise A Baur

The University of Sydney

\section{Research article}

Keywords: Obesity, Child, Health services, Tertiary care; Secondary care, Integrated health care, qualitative research, Models of care

Posted Date: September 27th, 2019

DOI: https://doi.org/10.21203/rs.2.15271/v1

License: (a) (i) This work is licensed under a Creative Commons Attribution 4.0 International License. Read Full License 


\section{Abstract}

Introduction For children with moderate to severe obesity, secondary and tertiary level services provide a platform for a more intensive approach toward weight management. Clinician and health care managers' (HCM) views are imperative for informing the nature of future services to treat such children.

Methods This concurrent mixed-methods study utilized questionnaires and semi-structured focus groups with clinicians, and one-on-one semi-structured interviews with $\mathrm{HCMs}$, who worked in six secondary or tertiary pediatric weight management clinics across five public hospitals in New South Wales, Australia. The Theoretical Domains Framework was employed to underpin the development of the interview guides. The recorded interviews were transcribed verbatim and coded line-by-line, noting the key emerging themes. The results from the questionnaire were analyzed using descriptive statistics.

Results Clinicians ( $\mathrm{n}=27$ ) provided multiple approaches to weight management, tailored to families and patients. Clinicians more often provided general healthy eating advice over a specific diet approach. Fourfifths used body mass index percentile change as a measure of success, as well as behavioral outcomes such as improvements in physical activity levels, screen time and sleep quality. Use of a combination of group and individual sessions were thought to improve engagement and reduce attrition rates. Clinicians and HCMs recommended integrating clinics into community centres and providing specific programs for sub-groups such as children from culturally and linguistically diverse communities or children with development delay. Many clinicians and HCMs stressed the importance of pediatric weight clinics using a holistic approach to treatment. Research into clinical outcomes for services was recommended to enable the development of a business case for further funding of pediatric weight management services.

Conclusion Addressing common barriers to current pediatric weight management services, and designing future models of care based on key stakeholders' preferences, are critical to achieving optimal care provision for this high risk population.

\section{Introduction}

Obesity is a major global public health problem. In Australia, one in four school-aged children have overweight and obesity, with $2 \%$ having severe obesity (equivalent to an adult body mass index [BMI] $>35$ $\mathrm{kg} / \mathrm{m}^{2}$ ) [1]. While prevention of obesity is important, so too is the provision of effective clinical care to those who are affected. Treatment of children with obesity requires multi-disciplinary, multicomponent interventions, with early parental/carer involvement to encourage family-wide changes [2-4]. The optimal model of care for children with obesity, from an economic and health viewpoint, is an evidence-based staged approach to management, where primary, secondary and tertiary services are involved depending on severity and the response to treatment [5]. In Australia, specialist multi-disciplinary programs exist mostly as secondary (healthcare services that do not have first contact with patient) or tertiary services (specialist services that usually require a referral from secondary care clinician) [6, 7]. For children with 
moderate to severe obesity, secondary and tertiary level care offers a more intensive, multi-disciplinary approach towards weight management [8].

There are few well-evaluated models of care for the treatment of pediatric obesity. There is a paucity of literature on the most effective clinical service models, in order to maximize both scalability and sustainability. In Australia, the literature on service models for pediatric obesity that does exist is based in primary care settings, rather than tertiary or secondary settings, or limited to a single health service or single model of care setting [9-11]. In practice, current service models vary markedly, with no clear referral pathways or frameworks for integration between primary, secondary and tertiary level services [12].

In New South Wales (NSW), the most populous state in Australia, several new secondary or tertiary weight management services were commissioned in 2017. The service models and health pathways for these new services drew on expertise from already established services, with variations based upon local patient needs, resourcing and clinical expertise. The implementation of these new services provided a unique opportunity to compare different models of care, albeit in some cases, at early stages of service development. There has been no research comparing clinician and healthcare manager (HCM) views on different service models for treating children with obesity. To date, research into clinician views on weight management has primarily focused on barriers to care, views on treating pediatric obesity and weight stigma only $[9,11,13]$. To address this gap in the literature, the aim of this study was to assess clinician and HCM views on models of care for pediatric weight management services in secondary and tertiary healthcare facilities.

\section{Methods}

\section{Study Design \& Setting}

A concurrent mixed-methods approach was employed utilising (i) questionnaires (stage 1), and (ii) semistructured focus groups with clinicians and one-on-one semi-structured interviews with HCMs (stage 2). A mixed-methods approach was chosen to ensure that viewpoints and perspectives obtained from participants were wide-ranging [14], and to account for the complexity of factors surrounding health research $[15,16]$. We published the protocol for the study [17] and based our report on the consolidated criteria for reporting qualitative research guidelines [18]. 


\section{Participants}

Recruitment took place between June 2018 and December 2018. Participants were clinicians who worked in six secondary or tertiary pediatric weight management clinics across five public hospitals in NSW, Australia. These clinics care for patients between the ages of 2-16 years, with one tertiary care service extending to adults. Each clinic uses a different service model, including a combination of face-to-face, telehealth and group education, with multi-disciplinary teams or dietitian-only clinics (Table 1). Clinicians were from a variety of health professions including medical, nursing and allied health. Eligible HCMs were those who had previously worked as clinicians (medical, nursing or allied health), and who now hold senior level positions in the organisations in which the pediatric weight management service was run.

Eligible clinicians and HCMs were identified by the head of the weight management service at each site. Clinicians were purposively sampled to ensure that a range of clinical experience and clinical backgrounds (nursing, medical and allied health) was represented [19]. Participants were sent an invitation to participate via email, and were provided with a study information sheet and consent form. Written consent was sought from the clinicians and verbal consent from HCMs participating in the oneon-one interviews. The study was approved by the Sydney Children's Hospitals Network Ethics Committee (Reference Number: LNR/17/SCHN/401).

\section{Data Collection and Analysis}

\section{Phase 1 Questionnaire}

The online clinician questionnaire was developed in collaboration with a multi-disciplinary team with expertise in obesity management, which included a pediatrician, dietitian and the project team. Five-point Likert scales were used to assess the clinicians' general management strategies ( 1 never to $5=$ always), dietary management ( $1=$ never to $5=$ always), specific management strategies ( $1=$ not important to $5=$ very important), and professional preparedness ( $1=$ =strongly disagree to $5=$ =strongly agree), with the questions based on a previously published survey [20]. Use of clinical outcome measures was assessed with yes/no responses across three age-groups: pre-school age, primary-school age and adolescent/high school age. Level of burnout was evaluated using the Masclach Burnout Inventory, a validated burnout scale [21], and weight bias using the Nutrition, Exercise and Weight Management Attitudes Scale [22]. The questionnaire also included clinician demographics. The final questionnaire was reviewed by the team of investigators for editing and pilot testing.

Participants were provided with a link to the survey to complete online. The clinical lead of each service completed additional questions on the clinic structure and referral process Data analysis was performed using SPSS statistical software (Version 24. 2012; IBM Corp., Armonk, NY). The Likert data for the general, nutrition and specific management questions were transformed into binary variables where "often" and "always" were combined (hereafter collectively referred to as "frequently"), and "never" and 
"rarely" were combined (hereafter collectively referred to as "infrequently"). For professional preparedness, the Likert data were transformed into binary variables, where "strongly disagree" and "disagree" were combined (hereafter collectively known as "disagree") and "agree" and "strongly agree" were combined (hereafter collectively known as "agree"). For the outcome measures, responses were treated as dichotomous variables where "yes" responses scored one point, while "no" responses were scored as zero.

\section{Phase 2 Interviews/focus groups}

Semi-structured focus groups were conducted with clinicians and semi-structured interviews were conducted with $\mathrm{HCMs}$ from each weight management service. The semi-structured interview guide for the focus groups and interviews was developed by a multi-disciplinary team of investigators including a pediatrician, dietitian and the project team, using an iterative process. A semi-structured interview guide was chosen as it allowed an exploration of both opinion and perceptions of the clinicians [23]. The development of the questionnaire and semi-structured interview guide was informed by the Theoretical Domains Framework (TDF) [24, 25]. The TDF was used to describe the influences of behavior change among clinicians working with children with obesity [24].

The focus groups aimed to explore the following: (i) establishment of the service; (ii) accessibility of the service; (iii) current processes used to manage patients; (iv) outcomes used to measure success; (v) clinician-perceived barriers and enablers to success in pediatric weight management and (vi) patient expectations for success. The HCM semi-structured interview guide aimed to explore the following: (i) health service views of pediatric weight management; (ii) future plans for the service; (iii) funding considerations; (iv) perceptions of strengths and weaknesses of the service and (v) recommendations for improvement. Both the clinician and HCM interview guide was reviewed and pilot tested by the research team and clinical leads of each weight management service.

All focus groups were facilitated by an experienced researcher with no prior relationship to participants who used the interview guide to aid discussion. The focus groups ranged in length from 60 to 90 minutes. The HCM interviews were conducted via telephone. Interviews ranged from 28 to 35 minutes in length. All interviews and focus groups were recorded and then transcribed verbatim. We performed qualitative data analysis using NVivo (version 12). An independent researcher (CS) coded interview and focus group data line-by-line, according to the relevant TDF domains determined by two researchers (JC, $\mathrm{CS}$ ). Key emerging themes from the data, within each of the theoretical domains, were also noted. Where data were relevant to more than one domain, we coded these into all relevant domains. We used qualitative data to aid interpretation of the quantitative findings from questionnaire data.

\section{Results}




\section{Participants}

A total of 27 clinicians and $15 \mathrm{HCMs}$ were invited to participate; all clinicians and $60 \%$ of $\mathrm{HCMs}$ agreed to participate. The clinicians were distributed across six weight management services. Dietitians represented the largest number (33\%), followed by pediatricians (19\%) and psychologists (19\%). Others who participated included nurses (15\%), physiotherapists (7\%), exercise physiologists (4\%) and medical fellows $(4 \%)$. The majority were female $(78 \%)$. The clinicians had a broad range of experience, with the median years practising as a clinician of 14 years (range: 1-38 years). The clinicians had worked with children with obesity for a median of 4 years (range: $0.5-26$ years).

The six weight management services had a variety of service models, ranging from a dietitian-led secondary level service to multi-disciplinary tertiary weight management services (Table 1). Two of the services only provided individual appointments with patients, three services provided a combination of group and individual sessions and one provided education in a group format only.

\section{Stage 1 Questionnaire}

\section{Management strategies}

Clinicians used a range of general management strategies. The most common advice given to families was related to physical activity, screen time and water (Table 2). The type of nutritional management strategies used varied, with clinicians tending to provide general healthy eating advice, in contrast to a specific diet approach. Almost $50 \%$ of clinicians frequently provided advice on intuitive/mindful eating [26] and a quarter reported always using a non-diet approach [27]. A large number of clinicians (80\%) reported using behavior modification techniques as part of their practice, although these were not specifically defined.

The most common type of dietary intervention was specific advice around reducing energy intake, with almost $80 \%$ of clinicians reporting that they frequently provided this type of dietary advice (Table 2 ). Almost one half regularly provided a specific meal plan to families. A total of $92 \%$ of clinicians reported that the most common reasons for choosing a specific dietary approach were patient preference and published research, with the least common reason being based on referral request ( $44 \%$ of clinicians).

\section{Training}

The majority of clinicians reported that they had adequate training to manage children with obesity, although just under half (48\%) felt that they could achieve successful outcomes (Table 2). Just over half of the clinicians indicated they had training in managing the co-morbidities seen in children with obesity. All of the medical practitioners and $75 \%$ of the nurses reported adequate training in managing children 
with obesity. In contrast, only $33 \%$ of allied health practitioners reported training. Sources of training included specialist training, online training modules and conferences.

\section{Outcome measures}

The majority of clinicians (80\%) used BMI percentiles as a measure of patient outcomes (Figure 1), with the clinical psychologists not doing so. Clinicians also commonly assessed other outcomes to track progress, such as the patient's physical activity levels, screen time and sleep quality. The frequency of these assessments did not differ between patient age groups. Clinicians were least likely to assess patient's depression scores or assess a patient's metabolic profile, although this was often done by at least one clinician at each site. None reported using triceps skinfold or mid upper arm circumference.

\section{Burnout}

Overall, the clinicians working in pediatric weight management services had low levels of burnout. The participants in this study reported low levels of emotional exhaustion (mean=9) and depersonalisation (mean=2) and high levels of personal accomplishment (mean=23). At an individual level, all participants reported low emotional exhaustion and high personal accomplishment, though $11 \%$ of participants reported a medium level of depersonalisation.

\section{Phase 2 Interviews/ Focus Groups}

Focus groups were undertaken with clinicians at each pediatric weight management service. A total of 27 clinicians participated in the focus groups and nine HCMs participated in one-on-one telephone interviews.

\section{Service development}

Each service was established quite differently (Table 3). Older clinics described their development as evolving slowly, with many iterations and varying resources. They described fronting many challenges as they evolved alongside the growing prevalence of childhood obesity and evidence-base in the literature. Newer clinics used the structure of more established services $(n=4)$ to inform their own development. Many decisions were guided by pragmatic considerations. For example, one clinic started with a simpler model, adapting the service thereafter to local demand using available resources. Each clinic consulted the scientific literature at set-up, with the majority of clinics revisiting the scientific literature every 2-3 years and adjusting service models as the evidence changed. One clinic however noted that its service model did not prioritize available evidence to inform its clinic structure or design. 


\section{Service structure and resources}

The waiting list for each clinic varied greatly, from no wait at all, to up to 6 months before patients could start the program. Clinicians noted several barriers restricting patient accessibility to the clinic, including limited access via public transportation ( $n=3)$, difficulties finding parking or expensive parking $(n=4)$, and inflexible clinic times $(n=4)$. The need for culturally and linguistically diverse (CALD) services, such as an interpreter, varied across regions. Some clinicians reported rarely having CALD patients present to the service, while others encountered them frequently.

Despite a focus on anthropometry at all services, participants stated that their focus was on sustainable changes in behavior and lifestyle rather than weight. Importantly, all clinicians placed emphasis on processes, rather than outcome goals. Process goals included improvements in comorbidities or medical outcomes $(n=2)$, behavioral and lifestyle changes $(n=5)$, patient education/awareness of obesity $(n=2)$ and overall patient and parent wellbeing $(n=2)$ and were perceived as more discernible and achievable than outcome goals such as weight change. Only one clinic believed that expectations weren't always clearly articulated to patients and could be better communicated at enrolment.

Routine patient data most commonly collected included weight $(n=4)$, height $(n=3)$, abdominal circumference $(n=4)$ and blood pressure $(n=3)$. Some clinics also regularly collected data on heart rate, cholesterol levels, medications and comorbidities, including behavioral and developmental diagnoses. Although not routinely done, for high risk patients attending the weight management services, clinicians organized blood collection $(n=3)$ to assess indicators such as fasting glucose and insulin, iron studies, liver enzymes and C-peptide. Some clinics $(n=3)$ also routinely measured the parents and/or siblings' heights and weights to normalize the process for the child and to facilitate discussion with the family about their own health.

\section{Service strengths and enablers of current models}

Clinicians noted many strengths of their services. These included having a multi-disciplinary team $(n=5)$, group $(n=4)$ and outdoor $(n=2)$ sessions and taking a holistic approach to weight management $(n=2)$. Less commonly implemented strengths included maintaining strong department and external links $(n=1)$, offering the clinic in a non-hospital location $(n=1)$ and using telehealth $(n=1)$. HCMs saw pediatric weight management services as an essential component of care delivered by their hospital, particularly to prevent comorbidities and chronic disease in adulthood. HCMs $(n=6)$ were also optimistic that funding would increase in the future, as the awareness of the importance of weight management in vulnerable groups was increasing, although one participant noted declining funds in the year prior.

HCMs noted several major strengths of current weight management services, including strong commitment, dedication and expertise from all clinic staff, as well as the hospital $(n=4)$, whole family 
involvement in treatment $(n=3)$, links to research centres, multi-disciplinary team approaches $(n=3)$, an evidence-based program $(n=2)$ and provision of a free service to families.

"[Treating obesity] needs someone with that passion and drive because it's not a quick fix. It has to have someone with that long-term view that we're going to keep going and we will get results"

\section{Service weaknesses and barriers of current models}

Several factors were felt by clinicians to lead to lower engagement among families and poorer attendance $(n=3)$. These included a lack of transport options $(n=1)$ and parking $(n=1)$, inflexible clinic times $(n=2)$, inadequate funding/resources $(n=4)$ and staffing deficits $(n=3)$. Particular staffing deficits were noted in the areas of administrative support, exercise physiology and social work. According to clinicians, patients and families from culturally and linguistically diverse (CALD) backgrounds, also faced additional and unique challenges $(n=3)$, including difficulty engaging with families, the inability to use interpreters in group sessions, a lack of culturally-appropriate resources and the unique needs of families from CALD populations

HCMs perceived barriers to the success of current models included limited program breadth meaning that not all affected children could be seen, a lack of recognition in parents that their child was above a healthy weight, the resource- and time-intensive nature of interventions, and low patient engagement.

"I think that patients and their families sometimes don't see [obesity] as a problem, or at least not as a medical problem, because it's kind of normal in a community to be overweight. So, any behavioral change.... is always going to be difficult"

One clinic attributed their poor attendance to inadequate follow-up as they divided their weekly clinic by younger and older age groups, meaning that patients were seen only once a fortnight. With clinics limited to just four locations in the state, HCMs noted that rural, regional and CALD patients faced additional barriers when accessing weight management services. In addition, two HCMs described a potential stigma associated with attending a weight management clinic.

"I even think that there's kind of a stigma attached to having children come to an acute facility because of their obesity... And probably it's kinder for that [family] to be [treated] in the community."

The challenges HCMs described were felt by the majority to be perpetuated by a lack of funding and resources $(n=7)$, especially for personnel, primarily dietitians, but also pediatricians, psychologists and exercise physiologists. Clinics that had dedicated clinicians, still had limited capacity as staff usually had to divide their time with other services. Insufficient resources also meant there was an inability to see all 
patients referred to the service, worsened by the lack of alternative services, or to continue care after discharge from the service.

\section{Health service perceptions of obesity and weight management}

HCMs unanimously agreed that obesity in childhood is a pressing issue and "a high profile, significant priority". Many noted the increasing awareness around the topic and the need to more proactively address weight management during childhood, given the future impact on individuals and on health system resources. There was less agreement among HCMs as to with whom the responsibility for pediatric weight management should lie. It was clear that primary, tertiary and community services all had a potential role to play. The majority of $\mathrm{HCMs}(n=6)$ endorsed an integrated, or shared care, approach between primary and specialist health services.

"If we don't have a seamless pathway between the different sectors of health, we will not maximize the benefit of this intensive and highly valued program that is offered."

The HCMs $(n=4)$ suggested that the treatment of children with overweight should lie with primary healthcare providers, while specialist or tertiary services should be reserved for the treatment of more severe obesity and its associated comorbidities. One HCM suggested that community services were preferable to hospitals for long-term weight management, given the difficulties patients may experience accessing hospitals. This difficulty in access could exacerbate poor attendance and discourage long term engagement. Some HCMs $(n=3)$ reported only seeing children with severe obesity, or feeling forced to prioritize these children due to long waitlists, resulting in a potential gap in care for those with less severe obesity, and in preventative services. $\mathrm{HCMs}(n=4)$ generally agreed that improvements needed to be made in initial discussions with families about a referral for their child to weight management services. Most $\mathrm{HCMs}(6 / 9)$ endorsed the need for additional training for hospital staff, particularly regarding navigating difficult discussions around a child's weight and weight-related health needs.

"[Patients] have to be the 95th percentile and above...to be able to gain this multi-disciplinary team input at the tertiary level.... but what we have lagged significantly is access to these lower level services ... looking at early detection, early intervention, early screening and identification."

\section{Recommendations for models of care}

The HCMs and clinicians had several concordant recommendations for an ideal model of care for future pediatric weight management clinics (Table 4). Some HCMs $(n=3)$ and clinicians $(n=2)$ suggested clinics run more frequently (i.e. at least weekly), with another form of contact (i.e. telephone, email or text message) in between, to promote maximal engagement. Most HCMs $(n=4)$ and clinicians $(n=3)$ endorsed a multi-disciplinary team, including a nurse, an occupational therapist, physiotherapist, social worker and 
a dedicated dietitian and psychologist. Both HCMs $(n=4)$ and clinicians $(n=2)$ discussed the importance of involving the whole family, for example by educating them and showing them how to cook affordable nutritional meals, particularly for families with low socioeconomic status. HCMs $(n=2)$ and clinicians $(n=3)$ also recommended a more integrated, or partnered, approach between services across the state. They recommended that each health district has its own service, with links to, and partnerships in, the community for prevention, intervention and follow-up.

\section{Discussion}

For children with moderate to severe obesity, secondary and tertiary level services provide a platform for a more intensive approach toward weight management [8]. Based upon the views of HSMs and clinicians taking part in this study, a holistic model of care that is multi-disciplinary, family-focused and provides frequent contact with patients through weekly clinics, was preferred by the majority. Clinicians reported using multiple approaches to weight management, tailored to families and patients. Attrition was noted to be a major challenge for services, and a combination of group and individual sessions was felt to be an effective strategy to manage this problem. Integrating clinics into community centres and providing specific programs for sub-groups, such as children from culturally and linguistically diverse communities or children with development delay, was also felt to be a necessary focus. The importance of embedding research into clinical practice to inform models of care and to enable the development of a business case for further funding of pediatric weight management services.

Consistent with previous recommendations for treating children with moderate to severe obesity, [3] clinicians and HCMs stressed that a multidisciplinary approach was a vital component of a pediatric weight management service. Varying professions made up the multi-disciplinary teams in our study. Services included doctors, dietitians, psychologists, physiotherapists and nurses. Given that lower socioeconomic status and family turmoil are independent risk factors for childhood obesity [28], the lack of social workers in most teams was identified as critical gap that should be addressed in the future. A majority of clinicians suggested using a family-centred approach in pediatric weight management services. Obesity is a chronic health condition that is intergenerational and many families have more than one member affected [29]. Furthermore, parents play a significant role in the provision of food in the household and parent modelling of healthy eating is a major factor in their children's food choices [30, 31]. Having pediatric weight management programs that provide education to the whole family is likely to improve outcomes for the child [10,32,33]. The literature suggests that families prefer services that provide a patient-and-family centred approach to education, tailored through the use of individualized information and resources[34][49]. Many centres were using motivational interviewing and assessment of stages of change with attending families. Motivational interviewing is effective for positive weight outcomes when delivered by primary care physicians [35] and pediatricians [36] and assessing stages of 
change may reduce attrition. For those with severe obesity, especially adolescent patients, a more personalized approach may be an essential part of the treatment plan in the future [37]. For younger patients, general eating and health advice along with teaching families intuitive eating may be as effective.

Clinicians and HCMs in our study reported concordant barriers to effective weight management, echoing previous research, in particular, the issue of patient attrition was highlighted.[38] Rates of attrition for pediatric weight management services have been reported as high as $42 \%$ [39], with up to $80 \%$ of families ceasing treatment early $[40,41]$. Reasons for attrition include logistical issues required to attending frequent appointments during work/school hours, the service experience not meeting family expectations [40], the length of follow-up [42], lack of weight loss [43], and the perceived stigma of attending a weight management clinic [39]. Some services in our study used initial group education to reduce long-term attrition rates, by clearly articulating treatment expectations and outcomes. Group education provides an opportunity to disseminate information in a less resource intensive way and identify families that are capable of attending a demanding weight management programe. Group education in combination with individual appointments may be more effective than individual therapy alone[4]. The group education sessions also appear to provide the added benefit of peer support for families, and may be one way of combating the perceived stigma of attending clinic by normalising the patient experience, and addressing social isolation $[44,45]$.

It was suggested in our study that community-based programs, linked to hospital-based clinics to allow more intensive management when needed, could reduce attrition in pediatric weight management services. Community programes could improve service accessibility and acceptability, including through avoiding the stigma associated with attending a hospital-based weight management service [34]. A framework for integrating primary care to secondary and tertiary services, including clear pathways for referral, would also be of benefit to ensure the provision of early, timely and appropriate care, including for those at a lower BMI.

Many HCMS and some clinicians highlighted the need to embed research into clinical practice in order to inform best-practice models of care and to enable the development of a business case for new and/or expansion of services. Clinical groups in Europe [46], Canada [47] and the USA [48] have developed central registries to record and analyse standard clinical outcome data from multiple sites in each country. These central registries are a way to produce high-quality and meaningful outcome data, which can be used to identify best practices for pediatric weight management and as a way of undertaking multi-site research [48]. These collaborations are thought to be an essential component of weight management and should be a focus for all pediatric weight management services in the future.

Strengths and Limitations 
This study was the first to assess clinicians' views on the barriers and enablers to pediatric weight management services across a number of different clinics and models of care. A strength was the addition of data from $\mathrm{HCMs}$ regarding the future resources that are likely to be provided to pediatric weight management services. The views of senior management in health services are more likely to influence decisions regarding funding for clinical services and their views are integral to future planning. A further strength was the assessment of views of clinicians working in a diverse range of pediatric weight management services, ranging from multi-disciplinary services that have been running for more than 10 years, to newly established dietitian-only clinics. However, given that three sites were newly established (< 2 years), they could not provide a more in depth perspective of the barriers and enablers to weight management. A follow-up study of these services would add value. Determining the views of $\mathrm{HCMs}$ whose centres did not have a pediatric weight management service in comparison to HCMs who work in services with pediatric weight management services could have strengthened the study. Our study focused on secondary and tertiary care clinicians who are currently working with children with obesity. It would be valuable to also elicit the views of pediatricians and clinicians in public and private settings who are not part of pediatric weight management services, but who provide education to children with obesity as part of their general clinical care. Assessing the views of parents and carers attending pediatric weight management services would also be valuable for informing models of care.

\section{Conclusion}

This study highlights the need for specialist pediatric weight management clinics to be multi-disciplinary, with a whole of family approach to weight management and that have the capacity for frequent followup (weekly). A social worker is likely to be an important member of a pediatric weight management team, but is often lacking. Initial group education with subsequent one-on-one appointments could be considered for specialist services to address attrition rates, which were noted to be a major barrier to care. Pediatric weight management services that include a community-based programe to promote accessibility and engagement are likely to be of value, as are stronger ties between different tiers of services. To strengthen the chance of future funding for pediatric weight management clinics and to optimize models of care, centres must embed research into their practice. The collection of standardized clinical data, within centralised data registries, should be viewed as usual practice.

\section{References}


1. Garnett SP, Baur LA, Jones AM, Hardy LL: Trends in the prevalence of morbid and severe obesity in Australian children aged 7-15 years, 1985-2012. PloS one 2016, 11(5):e0154879.

2. Altman M, Wilfley DE: Evidence update on the treatment of overweight and obesity in children and adolescents. Journal of Clinical Child \& Adolescent Psychology 2015, 44(4):521-537.

3. Dietz WH, Baur LA, Hall K, Puhl RM, Taveras EM, Uauy R, Kopelman P: Management of obesity: improvement of health-care training and systems for prevention and care. The Lancet 2015, 385(9a986):2521-2533.

4. Elvsaas I, Giske L, Fure B, Juvet LK: Multicomponent Lifestyle Interventions for Treating Overweight and Obesity in Children and Adolescents: A Systematic Review and Meta-Analyses. Journal of obesity 2017, 2017.

5. Kumar S, Kelly AS: Review of childhood obesity: from epidemiology, etiology, and comorbidities to clinical assessment and treatment. In: Mayo Clinic Proceedings: 2017: Elsevier; 2017: 251-265.

6. Spilchak PJ, Denney-Wilson E, King L, Baur LA: Tertiary paediatric obesity services in Australia. Journal of paediatrics and child health 2008, 44(5):243-247.

7. Cohen J, Brennan AM, Alexander S, Henderson J, Graham C, Baur LA: Assessment of Clinicians' Views for Managing Children with Obesity in the Primary, Secondary, and Tertiary Settings. Childhood Obesity 2019.

8. Barlow SE: Expert Committee Recommendations Regarding the Prevention, Assessment, and Treatment of Child and Adolescent Overweight and Obesity: Summary Report. Pediatrics 2007, 120(Supplement 4):S164-S192.

9. Barlow SE, Dietz WH: Management of child and adolescent obesity: summary and recommendations based on reports from pediatricians, pediatric nurse practitioners, and registered dietitians. Pediatrics 2002, 110(Supplement 1):236-238.

10. Visram S, Hall T, Geddes L: Getting the balance right: qualitative evaluation of a holistic weight management intervention to address childhood obesity. Journal of Public Health 2012, 35(2):246254.

11. Walker $\mathrm{O}$, Strong M, Atchinson R, Saunders J, Abbott J: A qualitative study of primary care clinicians' views of treating childhood obesity. BMC family practice 2007, 8(1):50.

12. Mihrshahi S, Gow ML, Baur LA: Contemporary approaches to the prevention and management of paediatric obesity: an Australian focus. Medical Journal of Australia 2018, 209(6):267-274.

13. Schalkwijk AA, Nijpels G, Bot SD, Elders PJ: Health care providers' perceived barriers to and need for the implementation of a national integrated health care standard on childhood obesity in the Netherlands-a mixed methods approach. BMC health services research 2016, 16(1):83.

14. Johnson RB, Onwuegbuzie AJ, Turner LA: Toward a definition of mixed methods research. Journal of mixed methods research 2007, 1(2):112-133. 
15. Morgan DL: Practical strategies for combining qualitative and quantitative methods: Applications to health research. Qualitative health research 1998, 8(3):362-376.

16. Shneerson CL, Gale NK: Using mixed methods to identify and answer clinically relevant research questions. Qualitative health research 2015, 25(6):845-856.

17. Cohen J, Alexander S, Critekos M, Garnett SP, Hayes AJ, Shaw T, Sim KA, Baur LA: The acceptability, effectiveness, and impact of different models of care for pediatric weight management services: protocol for a concurrent mixed-methods study. BMC health services research 2018, 18(1):417.

18. Tong A, Sainsbury P, Craig J: Consolidated criteria for reporting qualitative research (COREQ): a 32item checklist for interviews and focus groups. International journal for quality in health care 2007, 19(6):349-357.

19. Harris JE, Gleason PM, Sheean PM, Boushey C, Beto JA, Bruemmer B: An introduction to qualitative research for food and nutrition professionals. Journal of the American Dietetic Association 2009, 109(1):80-90.

20. Collins C: Survey of dietetic management of overweight and obesity and comparison with best practice criteria. Nutrition and Dietetics 2003, 60(3):177-184.

21. Maslach C, Jackson SE, Leiter MP, Schaufeli WB, Schwab RL: Maslach burnout inventory, vol. 21: Consulting Psychologists Press Palo Alto, CA; 1986.

22. Ip EH, Marshall MS, Vitolins M, Crandall SJ, Davis MS, Miller D, Kronner MD, Vaden MK, Spangler J: Measuring medical student attitudes and beliefs regarding obese patients. Academic medicine: journal of the Association of American Medical Colleges 2013, 88(2).

23. Louise Barriball K, While A: Collecting Data using a semi-structured interview: a discussion paper. Journal of advanced nursing 1994, 19(2):328-335.

24. Atkins L, Francis J, Islam R, O'Connor D, Patey A, Ivers N, Foy R, Duncan EM, Colquhoun H, Grimshaw JM: A guide to using the Theoretical Domains Framework of behaviour change to investigate implementation problems. Implementation Science 2017, 12(1):77.

25. Cane J, O'Connor D, Michie S: Validation of the theoretical domains framework for use in behaviour change and implementation research. Implementation science 2012, 7(1):37.

26. Dockendorff SA, Petrie TA, Greenleaf CA, Martin S: Intuitive Eating Scale: An examination among early adolescents. Journal of Counseling Psychology 2012, 59(4):604.

27. Clifford D, Ozier A, Bundros J, Moore J, Kreiser A, Morris MN: Impact of non-diet approaches on attitudes, behaviors, and health outcomes: A systematic review. Journal of nutrition education and behavior 2015, 47(2):143-155. e141.

28. Hemmingsson E: Early childhood obesity risk factors: socioeconomic adversity, family dysfunction, offspring distress, and junk food self-medication. Current obesity reports 2018, 7:204-209.

29. Moens E, Braet C, Bosmans G, Rosseel Y: Unfavourable family characteristics and their associations with childhood obesity: a cross-sectional study. European Eating Disorders Review: The Professional Journal of the Eating Disorders Association 2009, 17(4):315-323. 
30. Sahoo K, Sahoo B, Choudhury AK, Sofi NY, Kumar R, Bhadoria AS: Childhood obesity: causes and consequences. Journal of family medicine and primary care 2015, 4(2):187.

31. Cislak A, Safron M, Pratt M, Gaspar T, Luszczynska A: Family-related predictors of body weight and weight-related behaviours among children and adolescents: a systematic umbrella review. Child: care, health and development 2012, 38(3):321-331.

32. Sung-Chan P, Sung Y, Zhao X, Brownson R: Family-based models for childhood-obesity intervention: a systematic review of randomized controlled trials. Obesity Reviews 2013, 14(4):265-278.

33. Burchett HE, Sutcliffe K, Melendez-Torres G, Rees R, Thomas J: Lifestyle weight management programmes for children: A systematic review using Qualitative Comparative Analysis to identify critical pathways to effectiveness. Preventive medicine 2018, 106:1-12.

34. Perez AJ, Kebbe M, Holt NL, Gokiert R, Chanoine J-P, Legault L, Morrison KM, Sharma AM, Ball GD: Parent recommendations to enhance enrollment in multidisciplinary clinical Care for Pediatric Weight Management. The Journal of pediatrics 2018, 192:122-129.

35. Resnicow K, McMaster F, Bocian A, Harris D, Zhou Y, Snetselaar L, Schwartz R, Myers E, Gotlieb J, Foster J: Motivational interviewing and dietary counseling for obesity in primary care: an RCT. Pediatrics 2015, 135(4):649-657.

36. Davoli AM, Broccoli S, Bonvicini L, Fabbri A, Ferrari E, D’Angelo S, Di Buono A, Montagna G, Panza C, Pinotti M: Pediatrician-led motivational interviewing to treat overweight children: an RCT. Pediatrics 2013, 132(5):e1236-e1246.

37. Kelly AS, Marcus MD, Yanovski JA, Yanovski SZ, Osganian SK: Working toward precision medicine approaches to treat severe obesity in adolescents: report of an NIH workshop. International journal of obesity (2005) 2018, 42(11):1834-1844.

38. Skelton JA, Goff Jr DC, Ip E, Beech BM: Attrition in a multidisciplinary pediatric weight management clinic. Childhood Obesity 2011, 7(3):185-193.

39. Rhodes ET, Boles RE, Chin K, Christison A, Testa EG, Guion K, Hawkins MJ, Petty CR, Sallinen Gaffka B, Santos M: Expectations for Treatment in Pediatric Weight Management and Relationship to Attrition. Childhood Obesity 2017, 13(2):120-127.

40. Dhaliwal J, Nosworthy NM, Holt NL, Zwaigenbaum L, Avis JL, Rasquinha A, Ball GD: Attrition and the management of pediatric obesity: an integrative review. Childhood Obesity 2014, 10(6):461-473.

41. Zebracki K, Drotar D, Kirchner HL, Schluchter M, Redline S, Kercsmar C, Walders N: Predicting attrition in a pediatric asthma intervention study. Journal of Pediatric Psychology 2003, 28(8):519-528.

42. Spence ND, Newton AS, Keaschuk RA, Ambler KA, Jetha MM, Holt NL, Rosychuk RJ, Spence JC, Sharma AM, Ball GD: Predictors of short-and long-term attrition from the parents as agents of change randomized controlled trial for managing pediatric obesity. Journal of Pediatric Health Care 2017, 31(3):293-301.

43. Skelton J, Beech B: Attrition in paediatric weight management: a review of the literature and new directions. Obesity Reviews 2011, 12(5). 
44. Woolford SJ, Sallinen BJ, Schaffer S, Clark SJ: Eat, play, love: adolescent and parent perceptions of the components of a multidisciplinary weight management program. Clinical pediatrics 2012, 51(7):678-684.

45. Zenlea I, Thompson B, Fierheller D, Green J, Ulloa C, Wills A, Mansfield E: Walking in the shoes of caregivers of children with obesity: supporting caregivers in paediatric weight management. Clinical obesity 2017, 7(5):300-306.

46. Kurth B-M, Kamtsiuris P, Hölling H, Schlaud M, Dölle R, Ellert U, Kahl H, Knopf H, Lange M, Mensink $\mathrm{GB}$ : The challenge of comprehensively mapping children's health in a nation-wide health survey: design of the German KiGGS-Study. BMC Public health 2008, 8(1):196.

47. Morrison KM, Ball GD, Ho J, Mackie P, Buchholz A, Chanoine J-P, Hamilton J, Laberge A-M, Legault L, Thabane L: The CANadian Pediatric Weight management Registry (CANPWR): lessons learned from developing and initiating a national, multi-centre study embedded in pediatric clinical practice. $B M C$ pediatrics 2018, 18(1):237.

48. Kirk S, Armstrong S, King E, Trapp C, Grow M, Tucker J, Joseph M, Liu L, Weedn A, Sweeney B:

Establishment of the pediatric obesity weight evaluation registry: a National Research Collaborative for identifying the optimal assessment and treatment of pediatric obesity. Childhood Obesity 2017, 13(1):9-17.

\section{Tables}

Due to technical limitations, tables are only available as a download in the supplemental files section

\section{Figures}




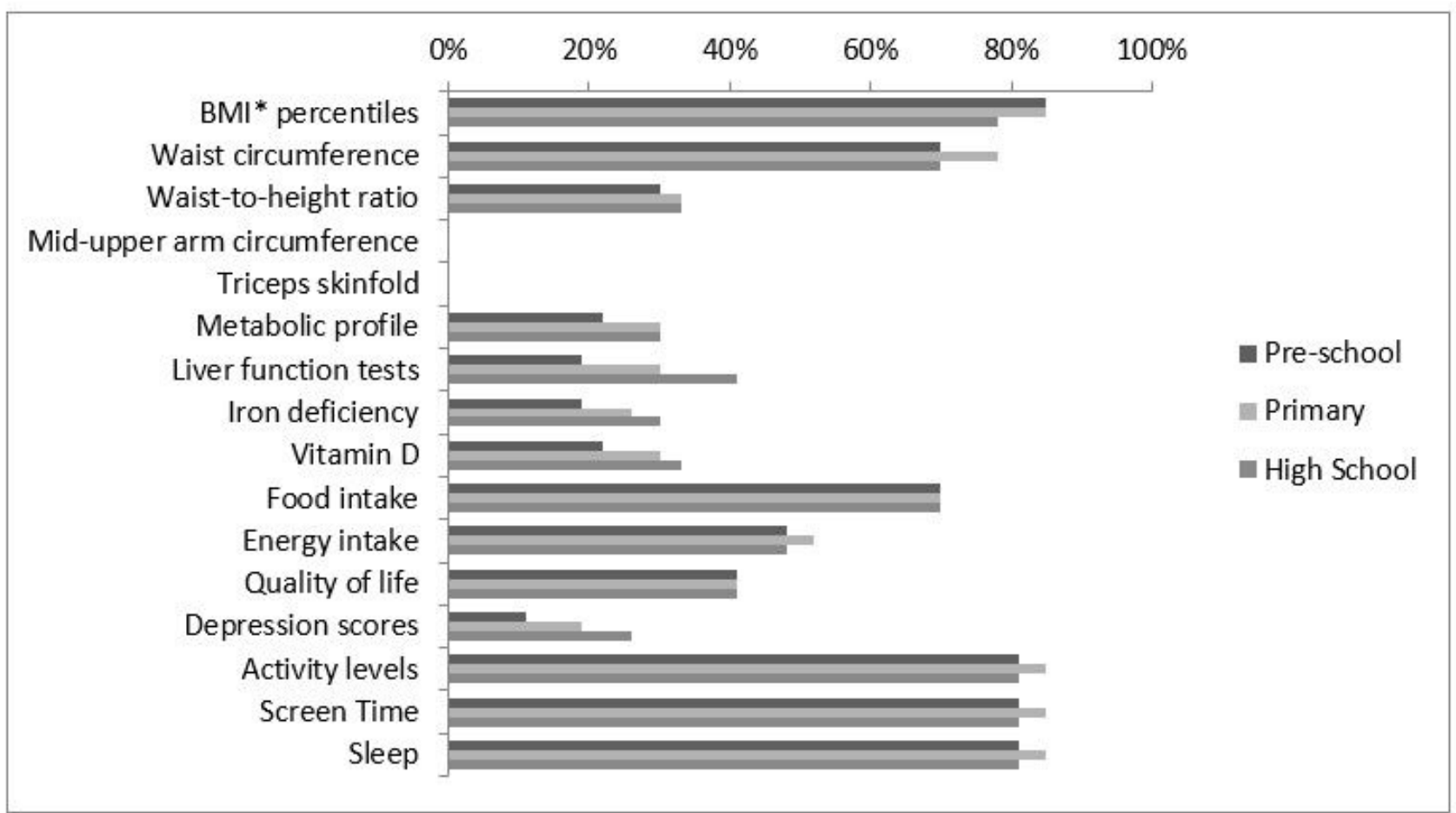

* BMI: Body Mass Index

\section{Figure 1}

Clinician reported outcome measures used for different age groups in the pediatric weight management clinics

\section{Supplementary Files}

This is a list of supplementary files associated with this preprint. Click to download.

- Table4ModelsofCareRecommendations.docx

- Table3ClinicianTDF.docx

- Table2Versionll.docx

- Table1Versionll.docx 